\title{
Dependence of AC Transport Current Losses of HTS Wires on Their Structures and Symthesizing Processes
}

\author{
Osami Tsukamoto, Daisuke Miyagi, Sadato Ishii and Naoyuki Amemiya \\ Faculty of Engineering, Yokohama National University. Yokohama. Japan \\ Satoshi Fukui \\ Faculty of Engineering, Niigata University, Niigata, Japan \\ Osamu Kasuu, H.li, Kaoru Takeda and Masatoyo Shibuya \\ Engineering Research Association for Superconductive Generation Equipment (Super-GM). Osaka. Japan \\ Masanao Mimura \\ The Furukawa Electric CO..LTD. \\ Kazuhiko Hayashi \\ Sumitomo Electric Industries. LTD. \\ Hisashi Yoshino \\ Toshiba Corporation
}

\begin{abstract}
HTS wires of various structures and synthesizing processes are being developed for power apparatus applications in Super-GM project. AC transport current losses of four of those wires were measured by an electric method using a lock-in amplifier. Measured wires are a $\mathrm{Bi} 2212$ rod fabricated by the laser pedestal growth method, a Y123 tape fabricated by the pulsed laser deposition and multilayered Bi2223/Ag sheathed wires of square and tape shapes. In this paper, based on measured data, dependence of AC transport current losses of HTS wires on their structures and synthesizing processes is discussed.
\end{abstract}

\section{INTRODUCTION}

High Temperature Superconductors (HTS) are more promising for AC power apparatuses such as power transmission cables, transformers, current limiters and motors than Low Temperature Superconductors (LTS) because efficiency of cooling AC losses is higher for HTS operating in $20 \mathrm{~K} \sim 77 \mathrm{~K}$ range than for LTS operating in $4.2 \mathrm{~K}$ range. The knowledge of $\mathrm{AC}$ losses and understanding of loss mechanism in HTS wires are important to apply HTS to the $\mathrm{AC}$ power apparatuses and develop low AC loss HTS wires and windings. The AC loss characteristics are influenced by many factors including configurations, structures, materials, synthesizing processes and critical currents of wires.

The R\&D on HTS wires of various structures and synthesizing processes for application to power apparatuses has been conducted in the Super-GM project [1]. We measured the $A C$ transport current loss characteristics of four kinds of wires of different structures and synthesizing processes which were developed in the Super-GM project.

Manuscript received September 14, 1998.

This work has been performed as a part of "R\&D on Superconducting Technology for Electric Power Apparatuses" under the New Sunshine Program of Agency of Industrial Science and Technology, MITI, being consigned by NEDO.
The losses were also measured in background DC magnetic field. The AC transport current losses were measured by measuring the voltage across the potential taps attached on the wire using a lock-in amplifier. The tested wires had various shapes, round, square and tape, therefore, proper care was taken for the potential leads arrangement. We used the spiral potential leads arrangement where the potential leads are spirally would on the cylindrical surface surrounding the wire. It has already been verified both theoretically and experimentally that the correct $\mathrm{AC}$ transport current losses can be measured using this arrangement regardless the shapes of the wires [2].

Measured $\mathrm{AC}$ losses were compared with theoretical values calculated from the Norris' formula based on the Bean model [3]. In this paper measured AC transport current losses are presented and the dependence of the AC loss characteristics on the wire structures and synthesizing processes are discussed by investigating the discrepancies between the experiment and theory.

\section{EXPERIMENTAL SET-UP AND}

The arrangement of the sample HTS wire is illustrated in Fig. 1. The sample shown in Fig. 1 was placed in a gap of a laminated iron core electromagnet. $\quad$ I is the transport current of the sample. The background field was parallel to the tape surface. The leads from the potential taps on the tape were spirally wound around the wire. This arrangement was also useful to measure the $A C$ transport current losses in the tight space of the magnet gap by avoiding problems caused by the limited space between the potential leads and the tape. The return current from the tape was adjusted to flow in the two divided current leads evenly and the leads were placed in the magnet gap to avoid the power flow between the power supplies of the sample and magnet by electromagnetic coupling. The AC transport current losses were measured by measuring the transport current and the resistive component of the voltage signal using a lock-in amplifier.

Specifications of the tested 4 wires, samples A, B, C and D 
are listed in Table I. The samples $A$ and $B$ are multilayered $\mathrm{Bi} 2223 / \mathrm{Ag}$ sheathed wires made by the multi-pipe method and shaped into tape and square forms respectively from round form wires [4]. The sample $\mathrm{C}$ is a Bi2212 rod fabricated by laser pedestal growth method [5]. The sample $\mathrm{D}$ is $\mathrm{YBCO}$ film deposited on textured $\mathrm{Ag}$ tape [6]. In the experiment $A C$ transport current losses of the samples wires were measured for different frequency with and without the DC background field.

\section{RESULTS AND DISCUSSIONS}

\section{A. Sample .A (Multilayered Bi2223 tape)}

Dependence of the critical current $I_{c}$ and $n$ value on the background field is shown in Fig. 2 (assuming the electric field along the wire is proportional to the $n$-th power of the wire current density). Fig. 3 shows the $A C$ transport current losses at $60 \mathrm{~Hz}$ dependent on the background field plotted against the peak value of the transport current $l_{p}$. In the figure $I_{p}$ is normalized by $I_{c}$ at the applied background field. The loss is expressed by the normalized value $\left(\mathrm{J} / \mathrm{m} /\right.$ cycle $/ \mathrm{A}^{2}$ : Joule per meter and cycle divided by $I_{c}{ }^{2}$ at the applied background field). In Fig. 3, the curve calculated from Norris' elliptical model is also plotted. Fig. 4 shows the dependence of the losses on the AC transport current frequency for various value of $i=I_{p} / I_{c}$ at $0 \mathrm{~T}$.

As seem in Fig. 3, the loss data deviate from the Norris' curve as the background field increases. Similar loss characteristics were observed in a multi filament Bi2223 / Ag sheathed tape [7][8]. Presently reasons for the discrepancy are not clear. It is considered partially because the $n$ value decreases as the back grand field increases (see Fig. 2) [8]. From Fig. 4, the losses slightly decrease as the frequency

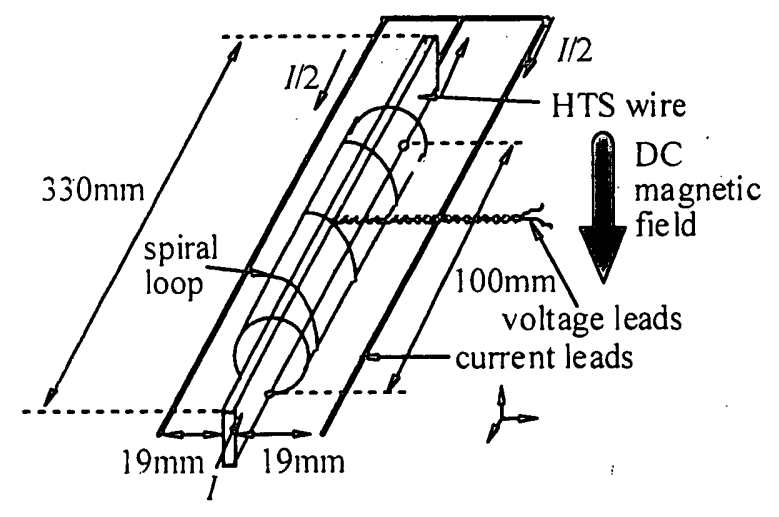

Fig. 1. Arrangement of sample wire.

TABLE I

SPECIFICATIONS OF SAMPLE WiRES

\begin{tabular}{|c|c|c|c|c|}
\hline Sample & $\mathrm{A}$ & B & $C$ & $\mathrm{D}$ \\
\hline Material & $\begin{array}{l}\text { Bi2223 } \\
\text { (Ag sheathed) }\end{array}$ & $\begin{array}{l}\mathrm{Bi} 2223 \\
\text { (Ag sheathed) }\end{array}$ & $\mathrm{Bi} 2212$ & $\begin{array}{l}\text { YBCO } \\
\text { (dep. Ag lape) }\end{array}$ \\
\hline Shape & Tape & Square & Rod & Tape \\
\hline $\begin{array}{c}\text { Dimension } \\
\qquad(\mathrm{mm})\end{array}$ & $3.87 \times 0.16$ & $0.81 \times 0.87$ & $\phi 5.6$ & $\begin{array}{l}9 \times 0.1 \\
(Y B C O \\
0.5 \mu \text { m thick) }\end{array}$ \\
\hline $\begin{array}{l}\text { I. (A) } \\
(1 \mu \mathrm{C} / \mathrm{cm}) \\
\mathrm{al} 0 \mathrm{~T} .77 \mathrm{~K}\end{array}$ & 38.1 & 2.1 & 283.5 & 2.1 \\
\hline
\end{tabular}

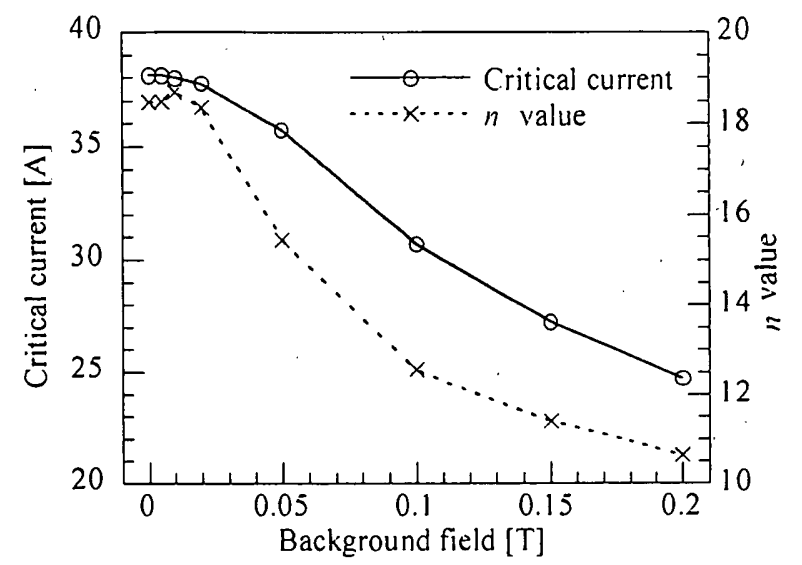

Fig. 2. Dependence of critical current and $n$ value on background field (Sample A : multilayered Bi2223 tape)

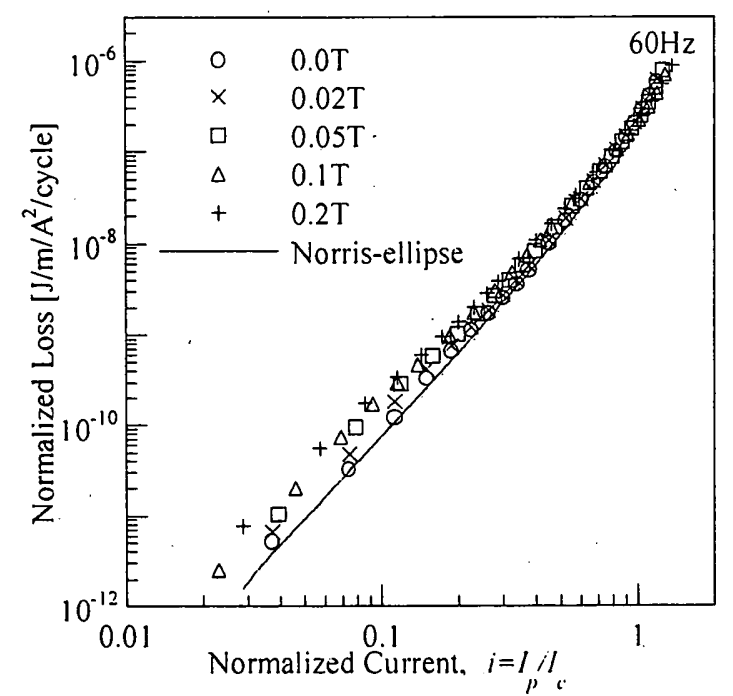

Fig. 3. Dependence of AC transport current losses on background field at $60 \mathrm{~Hz}$. (Sample A)

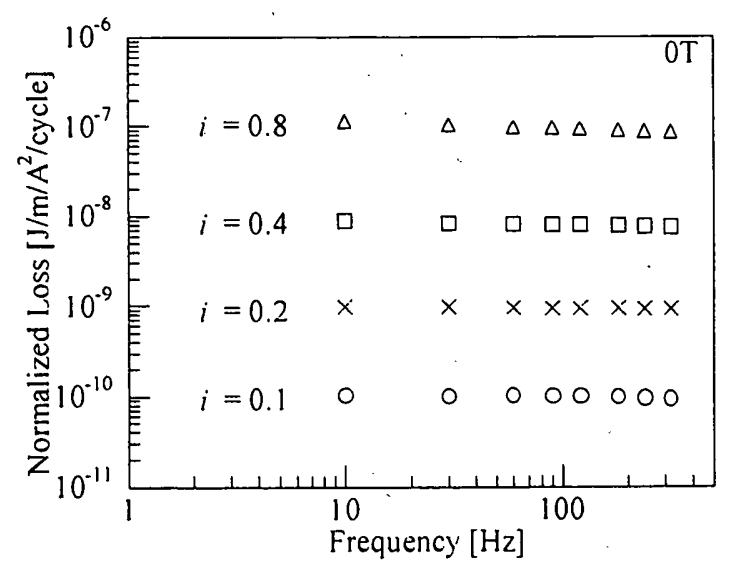

Fig. 4. Dependence of $A C$ transport current losses on frequency at $0 . T$. (Sample A)

increases. When $n=\infty$ (the Bean model holds in the wire); the losses are non-frequency-depend hysteresis losses. However, when $n$ is low, the losses tend to be lower at higher. frequency [8] and this is the case for the sample $A$. 


\section{B. Sample B (Multilayered Bi2223 square wire)}

Dependence of $I_{c}$ and $n$ value on the back ground field is shown Fig. 5, the AC losses dependent on the background field in Fig. 6 and the dependence of the losses on the

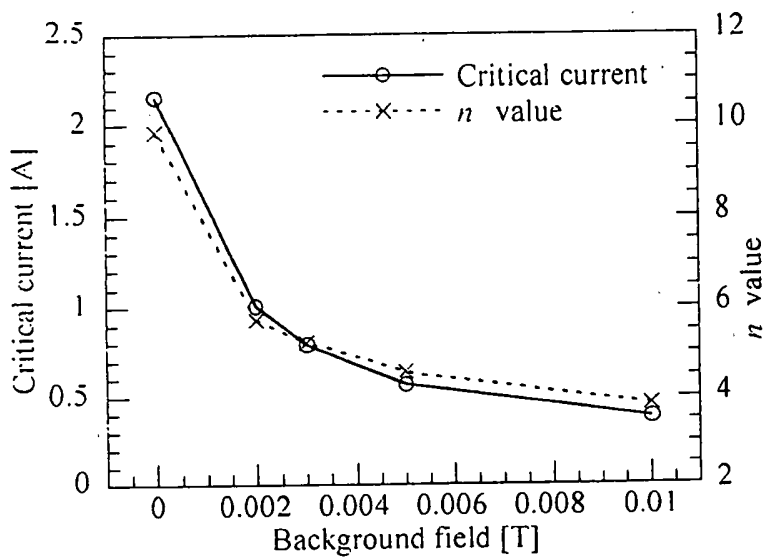

Fig. 5. Dependence of critical current and $n$ value on background field. (Sample B : multilayered Bi2223/Ag sheathed square wire)

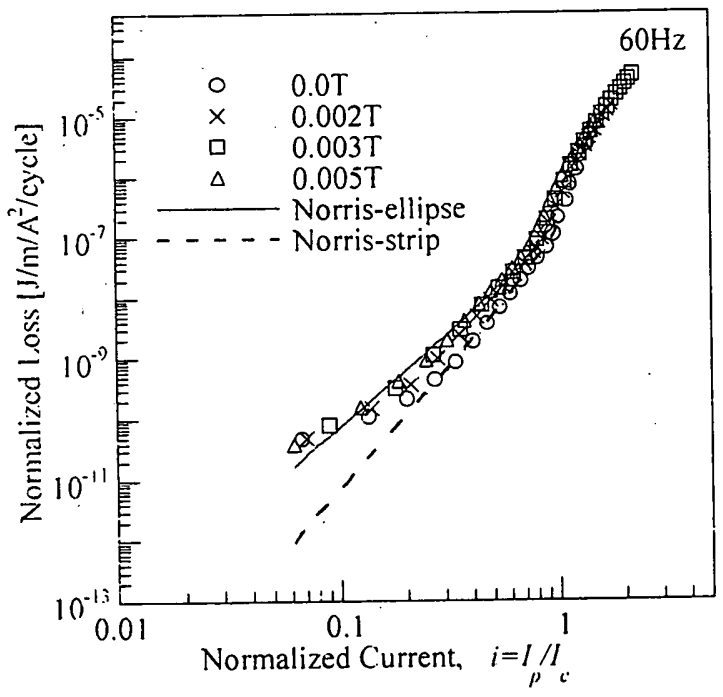

Fig. 6. Dependence of AC transport current losses on background field at $60 \mathrm{~Hz}$. (Samplc B)

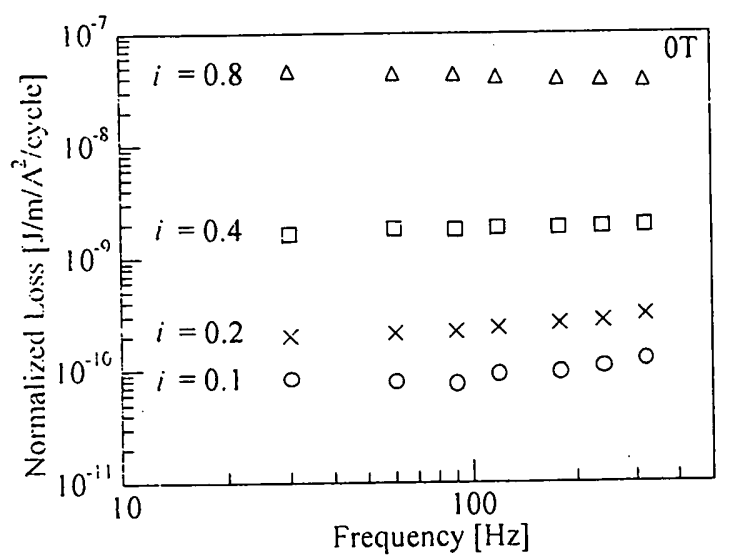

Fig. 7. Dependence of $A C$ transport current losses on frequency at $0 \mathrm{~T}$. (Sample B) frequency at $0 \mathrm{~T}$ in Fig. 7. Curves calculated by the Norris elliptical and strip models are also plotted in Fig. 6. As seen in Fig. 6, when the background field is zero, the measured losses follow the Norris' curve for the strip in the range $i=$ $0.3 \sim 1$. The losses depart from the strip model in the range $i$ $<0.3$ where the slope of the curve of measured data is more gentle than that of Norris' curve for the ellipse. When the background is applied, the loss data become closer to the Norris' curve for the ellipse. Detailed reasons for these loss behaviors are not clear. It is especially not clear why the loss data at $0 \mathrm{~T}$ follow the strip model in spite of the fact that the sample shape is square. At the moment, we considered those behaviors may be due to the inhomogeneous $J_{c}$ distribution across the wire cross section which changes with the background field.

\section{Sample C (Bi2212 rod)}

The sample $C$ had large $I_{c}$ and was difficult to test in the background field. Therefore the background field dependence was not measured. The normalized AC transport current losses at $0 \mathrm{~T}$ are plotted against $I_{p} / I_{c}$ for various frequency in Fig. 8.

The Norris curve in Fig. 8 is calculated from the elliptical (or round) model by assuming that the critical current density $J_{c}$ is uniform across the cross section of the rod. Slopes of the Norris' and measured data curves are same but the measured data is lower than the Norris' curve, which may be because $J_{c}$ is not uniform across the rod cross section. If $J_{c}$ of the outer part of the rod is higher than that of core part, the result shown in Fig. 8 can be explained [9]. Regarding to the non- uniformity of $J_{c}$ more studies are necessary. The losses decreased as the frequency increased, which may be caused by the relatively low $n$ value.

\section{Sample D (YBCO tape)}

Dependence of $I_{c}$ and $n$ value on the background field is shown in Fig. 9, the AC transport current losses dependent on the background field shown in Fig. 10 and the frequency

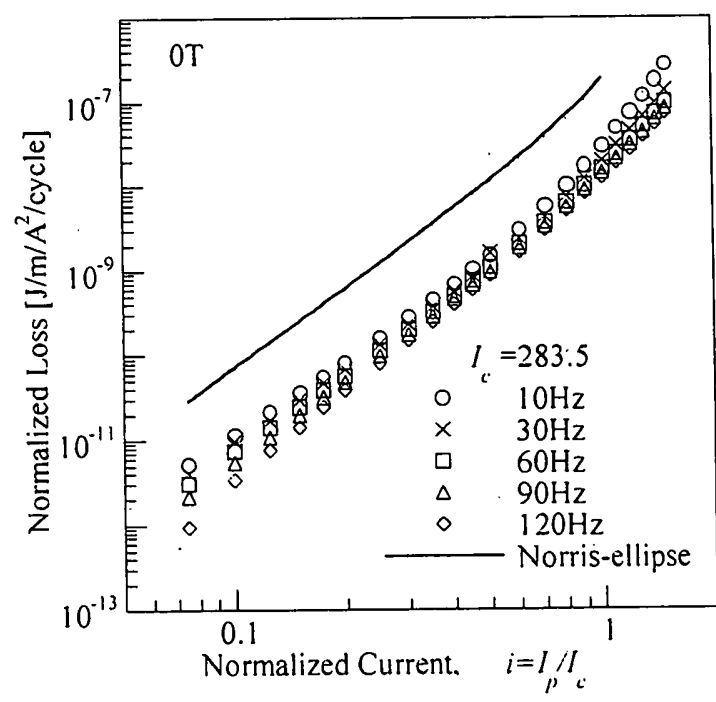

Fig. 8. AC transport current losses vs, $I_{n} / I_{c}$ for various frequency. (Sample C : Bi2212 rod) 
dependence of the losses at $0 \mathrm{~T}$ is shown in Fig. 11. In Fig. 10 , Norris' curves for elliptical and strip models are shown. As seen in Fig. 10, the loss data follow the strip model in the region of relatively high value of $i$ when the background field is zero and when the background field is applied, the data

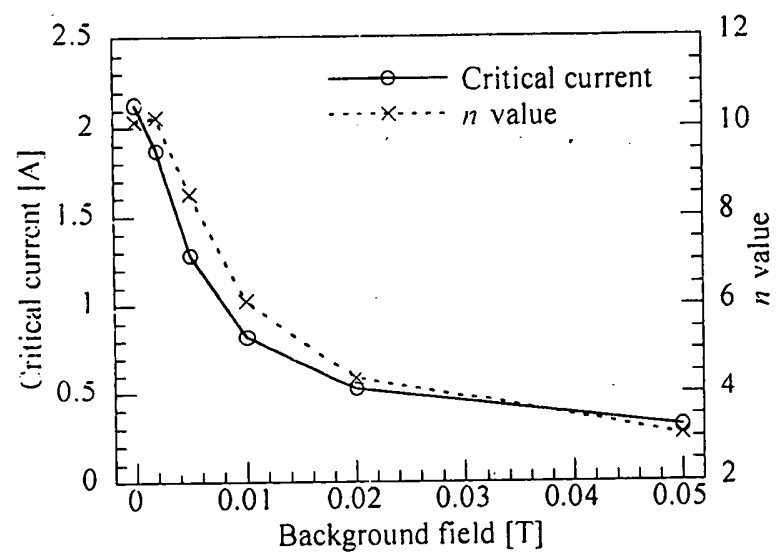

Fig. 9. Dependence of critical current and $n$ value on background field. (Sample D : YBCO tape)

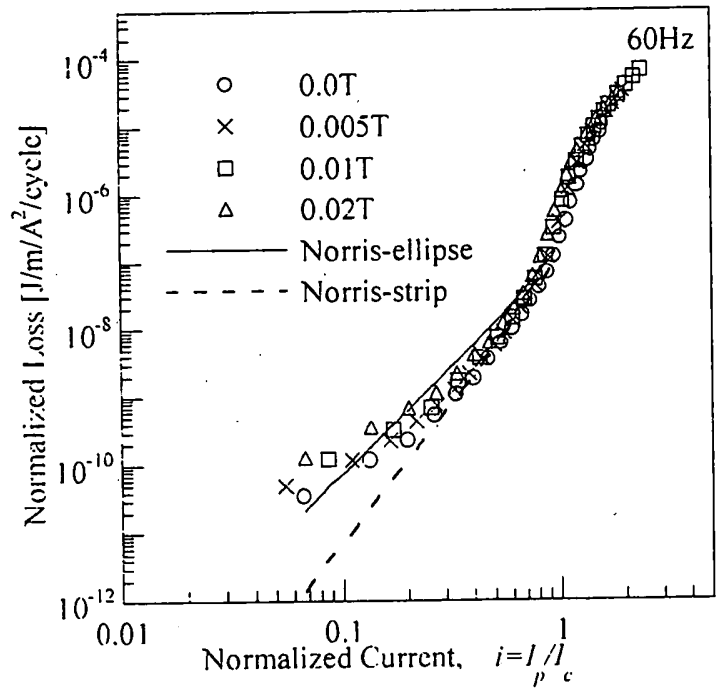

Fig. 10. Dependence of AC transport current losses on background field at 60 Hz. (Sample D)

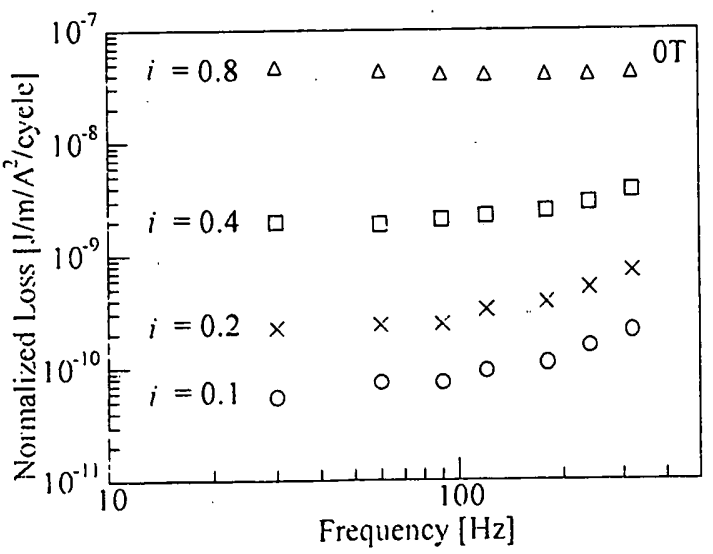

Fig. 11. Dependence of $A C$ transport current losses on frequency at $0 \mathrm{~T}$. (Sample D) move to the elliptical mode. In the region of relatively low value of $i$, the loss data depart from the strip model. As shown in Fig. 1] the losses increase as the frequency increases in the relative low $i$ region, while the losses slightly decrease as the frequency increases in the relatively high $i$ region. When the background field is not applied, it is considered the sample D, a thin film YBCO-deposited on Ag tape, has strip. like current distribution and that the magnetization losses due to the transport current follow the strip model basically. However, the eddy current loss component in the Ag base tape becomes apparent when $i$ and the magnetization loss become low. When the background field is applied, it is considered that the current distribution across the wire cross section. becomes elliptical.

\section{CONCLUSIONS}

The transport current AC losses of different structures and different materials have been measured. Their dependence on the frequency and DC background field has been studied also. The results are compared with the Norris' elliptical and strip models and it has been found that there were discrepancies between the experimental data and theoretical calculations. The discrepancies were different by the samples and can be explained by inhomogeneity of $J_{c}$ across the wire cross sections, the dependence of $n$ value on the background field and the effect of eddy current losses. Therefore, by studying the discrepancies, the loss mechanism of wires of various structures can be studied.

\section{REFERENCES}

[1] N.Yoshida, K.Sato, J.Yoshitomi and T.Ichikawa. "R\&D on oxide superconducting wires for application to power apparatuses," ICEC/ICMCI6

[2] S.Fukui, Y.Kitoh. T.Numata and O.Tsukamoto, "Transport Current AC. Losses of High-Tc Superconducting Tapes Exposed to AC Magnetic Field." presented at CEC/ICMC97.

[3] W.T.Norris. "Calculation of hysteresis losses in hard superconductors carrying ac: isolated conductors and edges of the sheets," J phys $D 3$, pp.489-507,1970.

[4] M.Mimura, K.Kosugi, A.Takagi, T.Watanabe, H.li, N.Uno, O.lkeda Y.Tanaka and K.Satou. "Fabrication of Conductor Using Bi-based Multilayered Wires." Advances in Supercoductivit' IX. vol. 2, pp.895898

[5] O.Kasuu, K.Takahashi, K.Sato and N.Yoshida, "Characterization of $\mathrm{Bi} 2 \mathrm{Sr} 2 \mathrm{CaCu} 2 \mathrm{Ox}$ Superconducting Rods and Tapes," Advances in: Supercoductivity: Vlll, vol. 2. pp.875-878

[6] M.Yamazaki, T.D.Thanh, Y.Kudo, H.Kubota, H.Yoshino and K.Inoue, "Preparation of in-plane aligned YBCO film on textured Ag tape," Advances in Supercoductivity: X. vol. 2. pp.619-622

[7] M.Ciszek, B.A.Glowacki. A.M.Campbell. S.P.Ashworth and W.Y.Loang. "Influence of External Magnetic Field and its Orientation on Transport" $\mathrm{AC}$ Losses in Bi-2223 and Tl-1223 Silver Sheathed Tapes," IEEE Trans. on Appl. Super..vol. 7. No. 2, June 1997.

[8] D.Miyagi, O.Tsukamoto. N.Amemiya. H.Mukai. K.Hayashi and K.Sato, "Influence of DC external magnetic field on AC transport current loss of of HTS tape." presented at ICMC98 Topical Conference on AC Loss and Stability of Low and High Tc Superconductor. Enschede, The Netherlands, to be published in Physica $C$.

19] F.Gomory and L.Gherardi. "Transport AC losses in round superconducting wire consisting of two concentric shells with different" critical current density," Physica C . pp.151-157, 1997 